\title{
Capacity-Optimal Training for Space-Time Modulation over a Time-Varying Channel
}

\author{
Christian B. Peel and A. Lee Swindlehurst \\ Brigham Young University \\ Electrical and Computer Engineering Dept. \\ 459 CB, Provo, UT 84602 \\ chris.peel@ieee.org, swindle@ee.byu.edu
}

\begin{abstract}
Using a model based on the time-autocorrelation function for time-varying MIMO channels, we find a lower bound on capacity for trained modulation, and find the training signal, training power, and length of training signal which maximize this bound. An approximation for the training frequency which maximizes the bound at high SNR is given for Jakes' fading model. The capacity for differential unitary space-time modulation is discussed and compared with that for trained modulation. We present several numerical examples to illustrate our results.
\end{abstract}

\section{INTRODUCTION}

Interest in time-varying multiple-antenna channels has increased recently, motivated by demand for high bandwidth mobile wireless data links. This has prompted investigation of techniques that can handle time varying channels. Trained and differential modulation are possibly the simplest of these methods. Though these techniques are not ideal for a timevarying channel, they are often used because of their simplicity. The parameters that optimize a lower bound on capacity for a training-based scheme have been found by Hassibi and Hochwald [1] for a Rayleigh distributed channel. They assume quasi-static fading, or that the channel coefficients are constant for a given coherence interval before changing to new independent values. The optimal training signal is found to be unitary, and the optimal training signal length, training power, and number of transmit antennas are also derived.

Another approach for time-varying fading is to assume that the receiver has no knowledge of the channel. This is in contrast to receivers for trained modulation, for example, which assume that the channel is known by the receiver. In the case of no channel knowledge, unitary signals have been found to be optimal [2]. Several signaling schemes have been developed using this assumption, including differential spacetime modulation [3], [4].

A different channel model was used in [5], [6] where the time-autocorrelation function of the channel coefficients and multiple first-order AR processes are used to model the channel. An effective SNR that includes the penalty due to channel estimation and time variation is found. In this paper, we use this channel model to derive a lower bound on capacity for training-based systems that use a minimum mean-square-error (MMSE) channel estimator. This bound is the extension of Hassibi and Hochwald's results [1] to a time-varying channel with known autocorrelation function. We find the training signal, training signal length, and training power which maximize the capacity bound, as well as an approximation to the optimal training period. We also discuss the capacity of differential modulation.

\section{Channel Model}

Assume a flat-fading communications environment with $M$ transmit and $N$ receive antennas. A complex channel coefficient that is assumed constant for a symbol of length $T_{s}$ describes the effect of the propagation between each pair of transmit and receive antennas. These channel coefficients are assumed to be independent from element to element across the antenna array. At each receive antenna, interference and other disturbances add temporally and spatially independent noise to the signal. In this case, the channel equation can be written in matrix form:

$$
X=\sqrt{\frac{\rho}{M}} S H+V,
$$

where $X$ is a $T_{s} \times N$ matrix of received data, $S$ is a $T_{s} \times M$ signal matrix, $H$ is a $M \times N$ matrix of channel coefficients, and $V$ is a $T_{s} \times N$ matrix of additive receiver noise. The matrices $S, H$, and $V$ all are assumed to be unit mean-square; ${ }^{1}$ the expected received SNR at each receive antenna is thus $\rho$. Though we will derive several initial results that do not depend on the distribution of $H$, the majority of this work will assume that $H$ has i.i.d. $\mathcal{C N}(0,1)$ entries.

\section{A. Channel Estimation}

We apply this fading model to both training and data transmission phases, resulting in the following joint channel model

$$
\left[\begin{array}{l}
X_{\tau} \\
X_{d}
\end{array}\right]=\left[\begin{array}{c}
\sqrt{\frac{\rho_{\tau}}{M}} S_{\tau} H_{\tau} \\
\sqrt{\frac{\rho_{d}}{M}} S_{d} H_{d}
\end{array}\right]+\left[\begin{array}{c}
V_{\tau} \\
V_{d}
\end{array}\right],
$$

where the training signal $S_{\tau}$ has dimension $T_{\tau} \times M$ and the data signal has dimension $T_{d} \times M$. Conservation of time and

\footnotetext{
${ }^{1}$ We say an $M \times N$ matrix $A$ has unit mean-square if $E\left[\operatorname{tr}\left(A A^{H}\right)\right]=$
} 
energy yields the following:

$$
\begin{aligned}
T & =T_{\tau}+T_{d} \\
\rho T & =\rho_{\tau} T_{\tau}+\rho_{d} T_{d},
\end{aligned}
$$

where $\rho_{\tau}$ and $\rho_{d}$ are the relative signal powers employed during training and data transmission, respectively. An important issue that we will address is the allocation of energy between training and data transmission. We use the parameter $\kappa$ to make the tradeoff:

$$
\begin{aligned}
\rho_{d} T_{d} & =\kappa \rho T \\
\rho_{\tau} T_{\tau} & =(1-\kappa) \rho T .
\end{aligned}
$$

The penalty due to channel estimation is also a major focus of this work. We consider the MMSE estimator:

$$
\hat{H}_{\mathrm{MMSE}}=\sqrt{\frac{\rho_{\tau}}{M}}\left(I_{M}+\frac{\rho_{\tau}}{M} S_{\tau}^{H} S_{\tau}\right)^{-1} S_{\tau}^{H} X_{\tau},
$$

and include the impact of using this channel estimator on the achievable capacity.

\section{B. Time Varying Model}

The quasi-static model of a time-varying channel assumes that $T$ is the coherence length of the channel, or that the channel is approximately constant for $T$ samples. In our model we go one step further, by allowing the channel to change between the data and training phases of transmission. This means that $T$ is no longer a coherence interval, but is still the coding interval or training period. We assume that the channel varies according to the following first-order auto-regressive (AR) or Gauss-innovations model:

$$
H_{d}=\sqrt{\alpha_{T}} H_{\tau}+\sqrt{1-\alpha_{T}} W_{d},
$$

where $H_{\tau}$ and $W_{d}$ have the same distribution, are zero-mean, and have unit mean-square; we also constrain $0 \leq \alpha_{T} \leq$ 1. Under this model, $H_{d}$ has the same distribution as $H_{\tau}$. Note that $\alpha_{T}=1$ produces a time-invariant channel, and $\alpha_{T}=0$ indicates a channel with no temporal correlation. It is important to note that the channel is not described by a single AR model, but rather with multiple first-order models, one for each time difference between the current sample and the reference channel.

The parameter $\alpha_{T}$ can be chosen to match the second order statistics of models based on the mechanisms of physical propagation. Let $r_{h h}(t)$ denote the time autocorrelation function of an element of $H_{d}$. Solving the Yule-Walker equations for $\alpha_{T}$ in the first-order AR process (6) we obtain

$$
\alpha_{T}=\left[\frac{r_{h h}(T / 2)}{r_{h h}(0)}\right]^{2},
$$

which provides a reasonable choice for $\alpha_{T}$. For example, assuming Jakes' model of the land mobile fading channel [7], $r_{h h}(t)=J_{0}(2 \pi f t)$, where $J_{0}(\cdot)$ is the zeroth-order Bessel function of the first kind, $f=f_{d} T_{s}, f_{d}$ is the maximum Doppler frequency in the fading environment, and $T_{s}$ is the sampling period. Under this model (7) leads to $\alpha_{T}=$ $J_{0}(\pi T f)^{2}$.
The Gauss-innovations model is an appropriate approximation when using decoders that depend only on a single reference channel (i.e., decoders that assume the channel is known). This fact is borne out by the simulation results of [5], where excellent agreement is obtained with data generated according to Jakes' model, but analyzed with the Gaussinnovations model.

\section{CAPACITY}

An analysis of the capacity achievable with trained modulation over the time-varying channel described above is now presented. A lower bound on capacity is derived, extending the work of [1] to a time-varying channel with known autocorrelation function. We find that while the lower bound is significantly different than that in [1], the parameters which optimize the bound are not. Finally, we present an analytic approximation for the best training frequency assuming the specific case of Jakes' model for the time-variations of the channel.

\section{A. Capacity and Effective SNR}

Section II describes in detail the relationship between the channel seen by the received and training data:

$$
\begin{aligned}
X_{d} & =\sqrt{\frac{\rho_{d}}{M}} S_{d} H_{d}+V_{d} \\
& =\sqrt{\alpha_{T} \frac{\rho_{d}}{M}} S_{d} H_{\tau}+\sqrt{\left(1-\alpha_{T}\right) \frac{\rho_{d}}{M}} S_{d} W+V_{d} .
\end{aligned}
$$

The difference between the estimated diffuse channel and that seen by the training data is $\tilde{H}_{\tau}=H_{\tau}-\hat{H}_{\tau}$. We can now separate the received signal from the effective noise, denoted by $V_{d}^{\prime}$ :

$$
\begin{aligned}
X_{d}= & \sqrt{\alpha_{T} \frac{\rho_{d}}{M}} S_{d} \hat{H}_{\tau}+ \\
& \underbrace{\sqrt{\alpha_{T} \frac{\rho_{d}}{M}} S_{d} \tilde{H}_{\tau}+\sqrt{\left(1-\alpha_{T}\right) \frac{\rho_{d}}{M}} S_{d} W+V_{d}}_{V_{d}^{\prime}} .
\end{aligned}
$$

The variance of the effective noise is now found:

$$
\begin{aligned}
\sigma_{V_{d}^{\prime}}^{2} & =\frac{1}{T_{d} N} E \operatorname{tr}\left[V_{d}^{\prime H} V_{d}^{\prime}\right] \\
& =\rho_{d} \alpha_{T} \sigma_{\tilde{H}_{\tau}}^{2}+\rho_{d}\left(1-\alpha_{T}\right)+1,
\end{aligned}
$$

where the variance of the channel estimation error is

$$
\sigma_{\tilde{H}_{\tau}}^{2}=\frac{1}{M N} E \operatorname{tr}\left[\tilde{H}_{\tau}^{H} \tilde{H}_{\tau}\right]
$$

There are several differences between the equations for $X_{d}$ in (2) and (8). In the former the channel contains an unknown diffuse component, while in (8) the channel component $\hat{H}_{\tau}$ is completely known. Theorem 1 of [1] provides a means to bound the capacity for trained modulation in this case. It indicates that the capacity for a training-based system with a known rotationally-invariant channel is lower bounded by 
a system with i.i.d. zero-mean Gaussian noise. By restricting $\hat{H}_{\tau}$ to have i.i.d. $\mathcal{C} \mathcal{N}(0,1)$ elements, we can write

$$
C_{\tau} \geq \frac{T-T_{\tau}}{T} E\left[\log \left|I_{N}+\rho_{\mathrm{eff}} \frac{\bar{H}^{H} \bar{H}}{M}\right|\right],
$$

where

$$
\rho_{\mathrm{eff}}=\frac{\rho_{d} \alpha_{T} \sigma_{\hat{H}_{\tau}}^{2}}{\rho_{d} \alpha \sigma_{\tilde{H}_{\tau}}^{2}+\rho_{d}\left(1-\alpha_{T}\right)+1}
$$

$\bar{H}$ is the normalized channel estimate $\bar{H}=\frac{1}{\sigma_{\hat{H}_{\tau}}} \hat{H}_{\tau}$, and

$$
\sigma_{\hat{H}_{\tau}}^{2}=\frac{1}{M N} E \operatorname{tr}\left[\hat{H}_{\tau}^{H} \hat{H}_{\tau}\right]
$$

\section{B. Optimizing the Lower Bound}

Inspection of (10) reveals that that the training signal only enters the equation through the effective SNR term. Thus, to maximize the lower bound on capacity, one need only maximize $\rho_{\text {eff. }}$. From the characteristics of our MMSE estimator, we know that $\sigma_{\tilde{H}_{\tau}}^{2}=1-\sigma_{\hat{H}_{\tau}}^{2}$. This allows a different form for $\rho_{\mathrm{eff}}$ :

$$
\rho_{\mathrm{eff}}=\frac{\rho_{d}+1}{\rho_{d} \alpha_{T} \sigma_{\tilde{H}_{\tau}}^{2}+\rho_{d}\left(1-\alpha_{T}\right)+1}-1
$$

Thus, minimizing $\sigma_{\tilde{H}_{\tau}}^{2}$ also maximizes (10); the problem of minimizing $\sigma_{\tilde{H}_{\tau}}^{2}$ has already been solved in [1] where the optimal training signal is found to satisfy

$$
S_{\tau}^{H} S_{\tau}=T_{\tau} I_{M}
$$

We will assume unitary training signals throughout the remainder of this work; in this case the variance of the channel estimate is

$$
\sigma_{\hat{H}_{\tau}}^{2}=\frac{\rho_{\tau} \frac{T_{\tau}}{M}}{1+\rho_{\tau} \frac{T_{\tau}}{M}},
$$

and the effective SNR becomes

$$
\rho_{\mathrm{eff}}=\frac{\rho_{d} \rho_{\tau} \alpha_{T} T_{\tau}}{\left(M+\rho_{\tau} T_{\tau}\right)\left(1+\rho_{d}\right)-\alpha_{T} \rho_{d} \rho_{\tau} T_{\tau}} .
$$

We now turn our attention to optimizing the power allocation between training and data transmission. Using (4) we write

$$
\rho_{\mathrm{eff}}=\frac{\alpha_{T} \kappa \rho \frac{T}{T_{d}}(1-\kappa) \rho T}{(M+(1-\kappa) \rho T)\left(1+\kappa \rho \frac{T}{T_{d}}\right)-\alpha_{T} \kappa \rho \frac{T}{T_{d}}(1-\kappa) \rho T} .
$$

Rewriting this equation to emphasize the relationship between $T_{d}$ and $M$, the following is obtained:

$$
\rho_{\mathrm{eff}}=\frac{\rho T}{T_{d}-M} \frac{\alpha_{T} \kappa(1-\kappa)}{-\kappa+\frac{T_{d}(M+\rho T)}{\rho T\left(T_{d}-M\right)}+\frac{\kappa(1-\kappa) \rho T\left(1-\alpha_{T}\right)}{T_{d}-M}} .
$$

We consider first the case $T_{d}>M$. Differentiating (16) and solving for $\kappa$ we find that

$$
\begin{aligned}
\kappa= & \frac{T_{d} \rho T+T_{d} M}{T_{d} \rho T-M \rho T}- \\
& \frac{\sqrt{T_{d}^{2} \rho T M+T_{d}^{2} M^{2}+M^{2} \rho T T_{d}+M \rho^{2} T^{2} T_{d}}}{T_{d} \rho T-M \rho T}
\end{aligned}
$$

The cases where $T_{d}=M$ and $T_{d}<M$ are handled in a similar way. Summarizing the results, we find that the best power allocation is

$$
\kappa=\left\{\begin{array}{cc}
\gamma-\sqrt{\gamma(\gamma-1)} & \text { for } T_{d}>M \\
\frac{1}{2} & \text { for } T_{d}=M \\
\gamma+\sqrt{\gamma(\gamma-1)} & \text { for } T_{d}<M
\end{array},\right.
$$

where

$$
\gamma=\frac{T_{d}(M+\rho T)}{\rho T\left(T_{d}-M\right)} .
$$

This is the same power allocation found in [1] where quasistatic fading was assumed.

We now consider optimizing the length of the training signal. Increasing $T_{\tau}$ increases the quality of the channel estimate, but decreases the amount of time available for data communication. Note that $T_{\tau}$ must be at least $M$ in order to obtain a channel estimate. Let $Q=\min (M, N)$ and $\lambda$ be an arbitrary eigenvalue of the matrix $\frac{\bar{H}^{H} \bar{H}}{M}$. Then the bound (10) can be expressed as

$$
C_{\tau} \geq \frac{Q T_{d}}{T} E \log \left(1+\rho_{\mathrm{eff}} \lambda\right) \triangleq C_{t}
$$

We choose to find the $T_{d}$ that will maximize $C_{t}$, and use that result to determine the best training data length. The derivative of $C_{t}$ with respect to $T_{d}$ is

$$
\frac{d C_{t}}{d T_{d}}=\frac{Q}{T} E \log \left(1+\rho_{\mathrm{eff}} \lambda\right)+\frac{Q T_{d}}{T} \frac{d \rho_{\mathrm{eff}}}{d T_{d}} E\left[\frac{\lambda}{1+\rho_{\mathrm{eff}} \lambda}\right]
$$

Assuming $T_{d}=M$, we obtain

$$
\rho_{\mathrm{eff}}=\frac{\rho^{2} T^{2} \alpha_{T}}{\rho^{2} T^{2}\left(1-\alpha_{T}\right)+4 M(M+\rho T)},
$$

and $\frac{d \rho_{\text {eff }}}{d T_{d}}=0$. This means that

$$
\frac{d C_{t}}{d T_{d}}=\frac{Q}{T} E \log \left(1+\rho_{\mathrm{eff}} \lambda\right) \geq 0,
$$

and making $T_{d}$ as large as possible, or $T_{\tau}$ as small as possible $\left(T_{\tau}=M\right)$, maximizes the lower bound $C_{t}$. The same result is obtained for $T_{d}>M$ and $T_{d}<M$. The results obtained to this point are now stated formally.

Theorem 1 (Optimal $S_{\tau}, \kappa$ and $T_{\tau}$ ): The training signal for the training-based scheme described in Section II that maximizes the lower bound (10) is unitary: $S_{\tau}^{H} S_{\tau}=M I_{M}$. The optimal training length is $T_{\tau}=M$, which implies that $T_{d}=T-M$. The optimal power allocation is given by

$$
\kappa=\left\{\begin{array}{cc}
\gamma-\sqrt{\gamma(\gamma-1)} & \text { for } T>2 M \\
\frac{1}{2} & \text { for } T=2 M \\
\gamma+\sqrt{\gamma(\gamma-1)} & \text { for } T<2 M
\end{array},\right.
$$

where

$$
\gamma=\frac{(T-M)(M+\rho T)}{\rho T(T-2 M)} .
$$

The corresponding effective SNR is given by

$$
\rho_{\text {eff }}=\left\{\begin{array}{cc}
\eta \alpha_{T} \frac{(\sqrt{\gamma}-\sqrt{\gamma-1})^{2}}{(\sqrt{\gamma}-\sqrt{\gamma-1})^{2}\left(1-\alpha_{T}\right) \eta+1} & \text { for } T>2 M \\
\frac{\rho^{2} T^{2} \alpha_{T}}{\rho^{2} T^{2}\left(1-\alpha_{T}\right)+4 M(M+\rho T)} & \text { for } T=2 M \\
\eta \alpha_{T} \frac{-(\sqrt{-\gamma}-\sqrt{1-\gamma})^{2}}{(\sqrt{-\gamma}-\sqrt{1-\gamma})^{2}\left(1-\alpha_{T}\right) \eta+1} & \text { for } T<2 M
\end{array}\right.
$$


where

$$
\eta=\frac{\rho T}{T-2 M} .
$$

The power allocation, training length, and training signal are the same as obtained in [1] for quasi-static fading, though the effective SNR (26) is significantly different.

The model presented in Section II allows the determination of the best training frequency, which depends on the power, number of transmit antennas, and autocorrelation function of the channel coefficients:

$$
\hat{T}=\underset{T}{\arg \max } \frac{T-M}{T} E\left[\log \left|I_{N}+\rho_{\text {eff }} \frac{\bar{H}^{H} \bar{H}}{M}\right|\right] .
$$

Because of the nature of the autocorrelation function and $\rho_{\text {eff }}$, a closed form solution for $\hat{T}$ is difficult to obtain. An approximation for the specific case of Jakes' model [7] can be found for the high-SNR case; similar approximations can be made for other autocorrelation functions. We begin by noting the high- $\rho$ effective SNR for this case:

$$
\hat{\rho}_{\text {eff }} \triangleq \lim _{\rho \rightarrow \infty} \rho_{\text {eff }}=\frac{\alpha_{T}}{1-\alpha_{T}} .
$$

This value may be approximated for small values of $f T$ by

$$
\hat{\rho}_{\mathrm{eff}} \approx \frac{2}{(f T \pi)^{2}} \triangleq \tilde{\rho}_{\mathrm{eff}} .
$$

For $f T=.05$,

$$
\frac{\tilde{\rho}_{\text {eff }}-\hat{\rho}_{\text {eff }}}{\hat{\rho}_{\text {eff }}}=0.01
$$

In other words, for $\rho>\hat{\rho}_{\text {eff }}$, and $T \leq 1 /(20 f)$ the relation (30) holds. A high-SNR, high-M capacity approximation to the capacity of a non-coherent system [8]

$$
C \approx\left(1-\frac{M}{T}\right) M \log \left(\frac{\rho}{e}\right)
$$

is valid for $M \leq N$, and $M \leq T / 2$. It is not immediately clear how high the SNR and $M$ must be; this is a topic for further research. In (32), the term $(1-M / T) M$ can be considered the number of degrees of freedom for this training-based scheme.

Using the high-SNR approximation (30) in place of $\rho$ in (32), differentiating, and solving for $T$, we obtain an expression for the best training period $\hat{T}$ to use at high SNR:

$$
\hat{T}=\operatorname{round}\left[M W_{0}\left(\frac{\sqrt{2 e}}{\pi M f}\right)\right],
$$

where $W_{0}(x)$ is the principal branch of Lambert's Wfunction [9] for any $x \in \mathcal{C}$, and gives the solution $w=W_{0}(x)$ such that $w e^{w}=x$. This approximation is restricted to the case of high SNR, high $M$, and $T \leq 1 /(20 f)$, and thus should be used with caution.

\section{DifFerential Modulation}

Differential modulation, like trained modulation, is designed for the case where the channel coefficients are unknown to both the transmitter and receiver. Because of this similarity, a comparison between differential and trained modulation is useful. The following theorem was shown in [6] to accurately characterize differential modulation.

Theorem 2 (Effective SNR for Differential Modulation): Given the data model of Section II, assume that the channel varies according to (6) with AR parameter $\alpha_{2 M}$. The effective SNR for differential space-time modulation is

$$
\rho_{D}=\frac{\rho \alpha_{2 M}}{1+\alpha_{2 M}+\left(1-\alpha_{2 M}\right) \rho} \leq \frac{\rho}{2} .
$$

In contrast to trained modulation, for differential modulation there is only one design parameter: the number of transmit antennas to use. For differential modulation with high numbers of transmit antennas $M$, a symbol (which has length $M$ ) is long enough that the channel has changed significantly from the previous symbol. The best number of antennas to use is not apparent: does the system designer use many antennas in order to obtain a high data rate, or fewer antennas so that the channel more accurately obeys the quasi-static model?

In order to answer this question fully, an expression for the capacity of differential modulation is required. However, this is an open problem [10], though there is some reason to believe that it is about half that for coherent modulation (when the receiver, but not the transmitter knows the channel). The unitary signals used for differential modulation have about half the degrees of freedom of an arbitrary signal matrix, leading to the idea that half the rate can be supported. In addition to this argument, we note that differential modulation can be seen as a member of the family of $T \times M$ rectangular unitary codes, for which the mutual information has been found [11]. Assuming $T=2 M$ and $N=M$, we find the capacity gain per $3 \mathrm{~dB}$ increase in SNR [8] to be

$$
\left(1-\frac{M}{T}\right) M=\frac{M}{2},
$$

which is half the gain seen for the corresponding coherent system.

For trained modulation the degrees of freedom seen is a function of the coding interval $T$, as well as the number of antennas used. If the system designer can chose $M$, then setting $M=T / 2$ maximizes capacity [1], [8], and results in $M / 2$ degrees of freedom, the same as for differential modulation! In this case, the high-SNR error floor for differential and trained modulation is also the same.

\section{NumERICAL EXAMPLES}

A few plots illustrate the preceding results. Figure 1 shows the capacity of trained modulation as a function of the power allocation factor $\kappa$. The vertical line at $\kappa=.71$ shows the best power allocation as determined from (24) for a normalized Doppler frequency of $f=0.003, M=N=2$ antennas, a training period of $T=14$, training length $T_{\tau}=M$, and an SNR of $\rho=20 \mathrm{~dB}$. The capacity bound from [1] $(f=0)$ is also shown.

Figure 2 shows results of Monte-Carlo simulations for the case of $M=N=4$ antennas, training length $T_{\tau}=M$, optimal power allocation $\kappa$, and $f=.003$. The inset shows that for the case of $30 \mathrm{~dB}$ SNR the best training period to use 


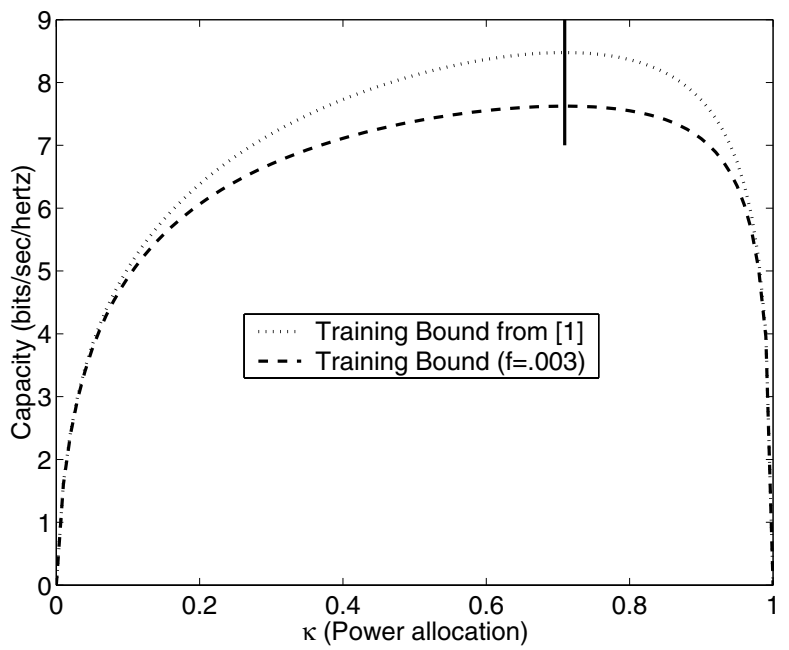

Fig. 1. Allocation of power between training and communication.

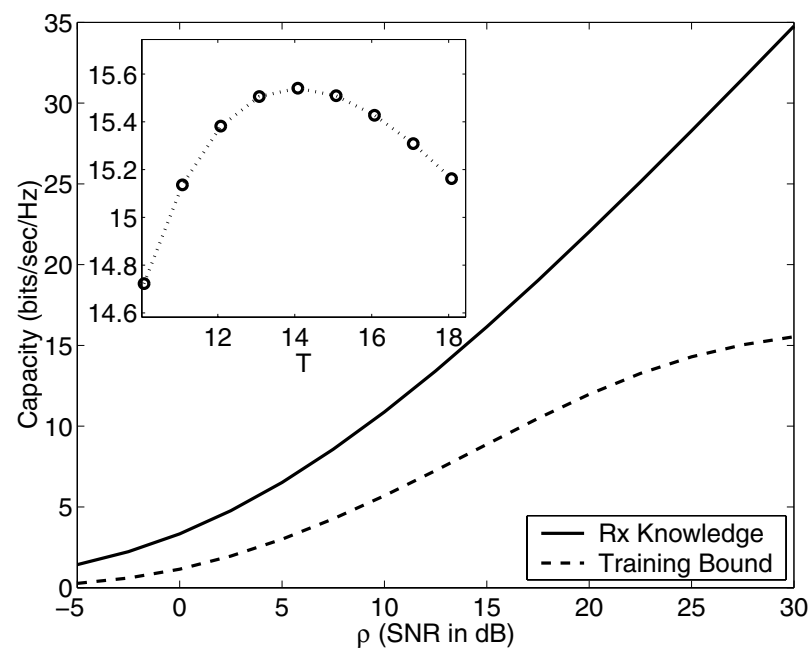

Fig. 2. Best training frequency.

is $T=14$. This is close to that obtained from the high-SNR approximation (33), which indicates $\hat{T}=12$. The main axis shows capacity as a function of SNR for trained modulation with $T=14$. The capacity when the receiver knows the channel is shown for comparison.

Figure 3 shows the effective SNR from (26) and from (34), as well as that from [1] for the case of $M=N=2$, training length $T_{\tau}=M$, optimal power allocation $\kappa$, coding interval $T=14$, and $f=.003$. In this case differential modulation has higher effective SNR than that for trained modulation. In contrast, differential modulation has $M / 2=1$ degree of freedom, while trained modulation has $(1-M / T) M=1.7$ degrees of freedom.

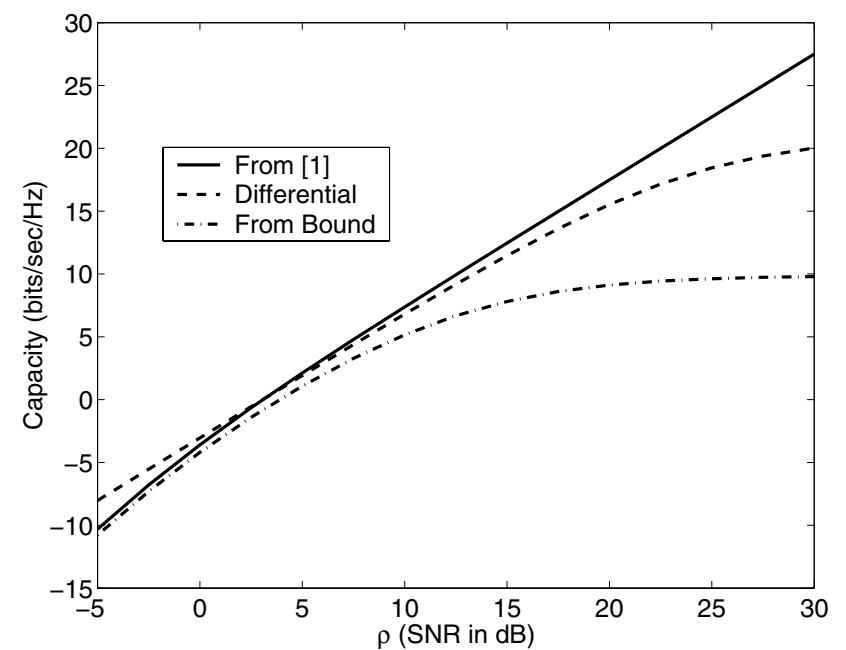

Fig. 3. Effective SNR.

\section{REFERENCES}

[1] B. Hassibi and B. M. Hochwald, "Optimal training in space-time systems," in Proceedings Asilomar Conference on Signals, Systems, and Computers, vol. 1, 2000, pp. 743-747, available from http://mars.belllabs.com/.

[2] T. L. Marzetta and B. M. Hochwald, "Capacity of a mobile multipleantenna communication link in Rayleigh flat fading," IEEE Transactions on Information Theory, vol. 45, no. 1, pp. 139-157, May 1999, available from http://mars.bell-labs.com/.

[3] B. L. Hughes, "Differential space-time modulation," IEEE Transactions on Information Theory, vol. 46, no. 7, pp. 2567-2578, November 2000, see http://www4.ncsu.edu/־blhughes/.

[4] A. Shokrollahi, B. Hassibi, B. M. Hochwald, , and W. Sweldens, "Representation theory for high-rate multiple-antenna code design," IEEE Transactions on Information Theory, vol. 47, no. 6, pp. 23352367, September 2001, available from http://mars.bell-labs.com/.

[5] C. B. Peel and A. L. Swindlehurst, "Performance of space-time modulation for a general time-varying Rician channel model," IEEE Transactions on Wireless Communications, Submitted, 2002.

[6] - "Analysis of space-time modulation in a time-varying channel," IEEE Transactions on Communications, Submitted, 2001.

[7] W. C. Jakes, Microwave Mobile Communications. IEEE Press, 1993.

[8] L. Zheng and D. N. C. Tse, "Communication on the Grassmann manifold: a geometric approach to the noncoherent multi-antenna channel," IEEE Transactions on Information Theory, vol. 48, no. 2, pp. 359-383, February 2002, see http://degas.eecs.berkeley.edu/ dtse/pub.html.

[9] R. Corless, G. Gonnet, D. Hare, D. Jeffrey, and D. Knuth, "On the Lambert W function," Advances in Computational Mathematics, vol. 5, pp. 329-359, 1996.

[10] T. Marzetta, "Two open problems in multiple-antenna communications," in Proc. DIMACS workshop on Signal Processing for Wireless Transmission, October 2002.

[11] B. Hassibi and T. L. Marzetta, "Multiple-antennas and isotropically random unitary inputs: the received signal density in closed form," IEEE Transactions on Information Theory, vol. 48, no. 6, pp. 1473-1484, June 2002. 